\title{
Statistical Mechanical Evaluation of Phase-Space Integrals.
}

\author{
F. LuRģat (*) and P. Mazur (**) \\ Institut des Hautes Etudes Scientifiques - Bures-sur-Ivette (S.-et-O.) \\ (Nuovo Cimento, 31, 140 (1964))
}

The formulae (4.24) and (4.25) should read:

$$
\begin{gathered}
G_{N}(0)=-(7 / 6) N^{-1}, \\
e \cdot r \cdot \gamma_{N}^{(1)}=e \cdot r \cdot \gamma_{N}^{(0)}\left[1-(7 / 6) N^{-1}\right] .
\end{gathered}
$$

In the formula (6.6) the denominator of the second term in the right-hand side should read $\beta P a$ instead of $\beta P b$. The formula thus reads:

$$
D(\beta)=\frac{c}{a^{2}}+6 \frac{3 \beta P+3 a-b}{\beta P a}+\frac{\beta P+a}{\beta^{2} P^{2} a}(15 a-6 \beta P)
$$

(*) Present address: Physique Théorique, Bâtiment 211, 91-Orsay.

(**) Present address: Instituut Lorentz voor Theoretische Natuurkunde, Langebrug 111, Leiden. 